\title{
Pulse EPR distance measurements to study multimers and
}

\section{multimerisation}

Katrin Ackermann and Bela E. Bode*

Biomedical Sciences Research Complex, Centre of Magnetic Resonance and

EaStCHEM School of Chemistry, University of St Andrews, North Haugh, St Andrews KY16 9ST, Scotland, UK

*Corresponding author. Email: beb2@st-andrews.ac.uk 


\title{
Pulse EPR distance measurements to study multimers and multimerisation
}

\author{
Pulse dipolar electron paramagnetic resonance (PD-EPR) has become a powerful \\ tool for structural biology determining distances on the nanometre scale. Recent \\ advances in hardware, methodology, and data analysis have widened the scope to \\ complex biological systems. PD-EPR can be applied to systems containing lowly \\ populated conformers or displaying large intrinsic flexibility, making them all but \\ intractable for cryo-electron microscopy and crystallography. Membrane protein \\ applications are of particular interest due to the intrinsic difficulties for obtaining \\ high resolution structures of all relevant conformations. Many drug targets \\ involved in critical cell functions are multimeric channels or transporters. Here, \\ common approaches for introducing spin labels for PD-EPR cause the presence \\ of more than two electron spins per multimeric complex. This requires careful \\ experimental design to overcome detrimental multi-spin effects and to secure \\ sufficient distance resolution in presence of multiple distances. In addition to \\ obtaining mere distances, PD-EPR can also provide information on \\ multimerisation degrees allowing to study binding equilibria and to determine \\ dissociation constants
}

Keywords: electron paramagnetic resonance; PELDOR; DEER; RIDME; multispin

\section{Introduction}

Pulse dipolar electron paramagnetic resonance (PD-EPR) is becoming increasingly important in structural biology [1,2]. The combination of methods such as pulsed electron-electron double resonance (PELDOR or DEER for double electron-electron resonance [3-5]) and DQC (double quantum coherence) filtered EPR [6] with sitedirected spin-labelling (SDSL) [7,8] has been frequently employed for precisely determining distances in-between paramagnetic centres in the range of 2 to $10 \mathrm{~nm}$ and beyond [9-11]. These long-range distances have been used to elucidate conformational changes $[12,13]$, assign conformational states $[14,15]$, or study quaternary structures of 
complex biological systems [16].

SDSL-PELDOR is the most established PD-EPR technique [1,2,10]. It is highly complementary to other biophysical and structural methods: i) it is a solution method that does not require (micro)crystallisation, ii) distances can be measured between identical labels, and iii) it is not easily overwhelmed by the complexity of the system under study, since commonly only the introduced spin labels result in EPR signals. Thus, it can be applied to intrinsically disordered or flexible systems, or can be used to target lowly populated conformational states [17].

The dipolar couplings measured in PD-EPR are usually much smaller than the inhomogeneous EPR line width. In PELDOR this small coupling frequency is recovered by refocusing all other inhomogeneities during formation of a Hahn echo that is subsequently refocused. The set of spins contributing to the refocused echo is often called A-spins. The dipolar coupling is selectively introduced by inverting a set of Bspins with a microwave pulse of different frequency at a specific time $t$ during the evolution of the transverse A-spin magnetisation. The A-spins will now accumulate a phase factor of $\omega_{\mathrm{D}} t$ with $\omega_{\mathrm{D}}$ being the distance- and orientation-dependent dipolar coupling (eq 1) $[5,18]$.

$$
\begin{gathered}
\omega_{D}=2 \pi D_{\text {dip }}\left(1-3 \cos ^{2} \theta_{A B}\right) \\
D_{\text {dip }}=\frac{\mu_{0} \mu_{B}^{2} g_{A} g_{B}}{4 \pi \hbar} \frac{1}{r_{A B}{ }^{3}}
\end{gathered}
$$

$\hbar$ is the reduced Planck's constant, $\mu_{0}$ the permeability of vacuum, $\mu_{\mathrm{B}}$ is the Bohr Magneton, $g_{\mathrm{i}}$ is the $g$-factor of spin i, $r_{A B}$ is the scalar distance between the two spins and $\theta_{\mathrm{AB}}$ is the angle between the distance vector and the external magnetic field. Spins A and B can be of the same chemical nature. However, the same spins must not be 
excited by both microwave frequencies. Ideally, the two different resonance frequencies are caused by different orientations or different nuclear spin states of coupled nuclei. The A-spin echo will be modulated by a $\cos \left(\omega_{\mathrm{D}} t\right)$ term for pairs in which the B-spin was inverted by the pump pulse. Thus, the overall modulation and the effect-sensitivity in a two-spin system $V_{\text {intra }}$ (eq 2) will scale linearly with the fraction of B-spins inverted by the pump pulse (inversion probability $\lambda$ multiplied by labelling degree $f$ ) $[10,19]$.

$$
V_{\text {intra }}=1-\lambda f\left(1-\cos \left(\omega_{D} t\right)\right)
$$

Consequently, great efforts have been taken to maximise the number of inverted B-spins and thus sensitivity. The opportunity to use high power at 34 and $95 \mathrm{GHz}$ has led to breakthroughs for the sensitivity of Q- and W-band PELDOR, respectively. Sensitivity demands a finite concentration for real samples leading to a background decay determined by dipolar coupling to spins in different complexes (eq 3).

$$
\begin{gathered}
V=V_{\text {intra }} \times V_{\text {inter }} \\
V_{\text {inter }}=\exp (-k t)
\end{gathered}
$$

$\mathrm{k}$ depends on the sample concentration and the inversion probability $\lambda[20]$. In homogeneous samples, this background $V_{\text {inter }}$ can be approximated to a monoexponential decay. However, the isolation of $V_{\text {intra }}$ from the overall signal bears higher uncertainties if the maximum dipolar evolution time $t_{\max }$ is not much larger than the inverse dipolar coupling. 
In multimers or other systems bearing $N$ multiple spins within one complex the signal factorises over all possible B-spins (eq 4) with the total modulation depth $\Delta$ (eq 5) $[21,22]$.

$$
\begin{aligned}
& V_{\text {intra }, N}=\frac{1}{N} \sum_{A=1}^{N} \prod_{B \neq A}^{N} V_{\text {intra }} \\
& \Delta=1-(1-\lambda f)^{N-1}
\end{aligned}
$$

For PELDOR, the most commonly used spin labels for SDSL are stable nitroxide radicals such as MTSL ((1-oxyl-2,2,5,5-tetramethyl- $\delta 3$-pyrroline-3-methyl) methanethiosulfonate) $[7,23]$. However, paramagnetic metal ions that can be used in combination with systems bearing a native or introduced metal-binding site, are receiving increasing attention [24-27]. These labels are more stable than nitroxides in the reducing environment of the cell $[28,29]$, and are especially attractive in combination with evolving methodology, such as shaped pulses [30,31] or RIDME (relaxation-induced dipolar modulation enhancement) [32], to compensate for the reduced overall modulation depth $\Delta$ achievable with metal ions compared to nitroxides. Compared to PELDOR, fast relaxation times and broad spectra (as observed in metal ions) can be less problematic using RIDME. While PELDOR is by far the more established and better understood technique, RIDME has great potential, and recent advances such as the dead-time free five-pulse version [33], averaging schemes to reduce nuclear modulation artefacts [34], or modified Tikhonov kernels to eliminate overtones [35], will significantly widen the scope of this method.

Pulse EPR distance measurements are especially attractive in combination with known sub-structures obtained from complementary methods such as NMR or crystallography $[15,36]$. This approach allows for example conformational studies on 
membrane proteins to identify "hot spots of motion", i.e. protein regions undergoing major conformational changes, in cases where not each functional conformation can be structurally characterised by crystallography or cryo-electron microscopy. Notably, already before pulse EPR distance measurements came into focus, continuous wave (CW) EPR measurements were performed on membrane proteins [37]. These $\mathrm{CW}$ experiments provided information on accessibility [38], dynamics [8], polarity [39], or short inter-spin distances (up to $2 \mathrm{~nm}$ ) [40].

Here, we will focus on pulse EPR applications to multimeric systems. Quantitative modulation depth studies using PELDOR and RIDME are performed mainly on dimeric systems, and multimeric systems such as membrane proteins are used in combination with the PELDOR technique for accurate extraction of nanometre distances.

\section{PELDOR on multi-spin systems}

\section{Multi-spin effects}

The presence of more than two spins per protein complex or quaternary structure results in an increase in total modulation depth (eq 4) [21] and in combination frequencies leading to so-called multi-spin effects [19,21]. These result from sum and difference frequencies in the dipolar oscillations and without extra treatment they often lead to erroneous distance distributions. So-called 'ghost peaks' [41] in the distance distribution that do not correspond to a true distance and the loss of the true intensities of populations with long distances have both been attributed to combination frequencies $[41,42]$. Distance distributions are generated from the primary data by solving a moderately ill-posed inverse problem. This is most frequently done by Tikhonov regularisation to ensure a smooth distance distribution. The Kernel attributes a Pake 
distribution of frequencies to every distance based on a two-spin system [43,44], and modification of the Kernel to allow for multiple spins is not trivial. Combination frequencies will depend on correlations between distances and between dipolar angles as well as the probability of multiple excitation. Commonly, the two-spin Kernel is used approximatively even in multi-spin systems $[41,42]$.

Multi-spin effects arising from data inversion by use of the two-spin Kernel have been shown to worsen with the number of spins present per complex and can severely hamper data analysis and interpretation $[42,45]$. However, multimeric proteins and their conformational changes during function are an important target in structural biology and are also increasingly studied by pulse EPR.

\section{Membrane proteins}

Membrane transporters and channel proteins are often homo-multimers playing essential roles in cellular signalling pathways, and many of them are potential drug targets [46]. Membrane proteins are notoriously difficult to crystallise, and the ability for investigating conformational changes and functional dynamics makes them highly attractive targets for EPR spectroscopy [46]. Both, pulse EPR methods and CW EPR have been used extensively to investigate membrane proteins in a large variety of biological systems [7,15,40,47-61]. However, for higher multimers multi-spin effects have been demonstrated to seriously affect PELDOR distance distributions obtained from multimeric membrane proteins with nitroxide spin labels attached to each monomer [45]. This has even led to the coerced disregard of all but the shortest distance for the sake of unambiguity in data interpretation [15,51]. Additionally, limitations in the achievable dipolar evolution time can result in low distance resolution and higher uncertainties for the longer distances present in the system [62]. Generally, higher uncertainties in labelling efficiency have been observed for transmembrane sites, and 
nearby lipid-binding sites can affect binding of the spin label [14].

\section{Suppressing multi-spin effects}

\section{Power-scaling}

Recently, a post-processing approach for reducing multi-spin effects has been implemented in DeerAnalysis, the gold standard of PD-EPR data analysis [44]. In this approach, the background-corrected time trace is scaled to the power of $1 /(N-1)$, where $N$ is the number of spins in the system [41]. The approach was validated on chemical model systems containing up to three spins and simulated systems with up to five spins [41]. However, we could demonstrate that in systems with seven or more spins powerscaling alone is no longer sufficient to fully suppress multi-spin effects at X-and Qband frequencies ( $~ 9$ and $34 \mathrm{GHz}$ ), if still the maximum achievable power is applied ( $\lambda_{0}$, see below) [45]. Nevertheless, power-scaling is a highly useful approach that is easy and practically free to implement during data analysis as it does not require modified experimental settings or changes in sample preparation. Importantly, in cases where the number of spins (or the oligomeric state) in the system is unknown, powerscaling of the PELDOR traces can help to confirm the presence of multi-spin effects, i.e. if the distance distribution changes with power-scaling it is likely that multiple spins per protein complex are present.

\section{$\lambda$-reduction}

An experimental approach to reduce multi-spin effects is to reduce the probability of simultaneous excitation of B-spins by the microwave pump pulse, and can thus no longer contribute to the creation of multi-spin effects. The maximum achievable probability of flipping spins, $\lambda_{0}$ (or inversion efficiency [19]), is dependent on the 
experimental hardware, and can be reduced by modifying the pump pulse in a PELDOR experiment [19].

Recently, we evaluated the combined use of $\lambda$-reduction and power-scaling in a heptameric membrane protein and were able to demonstrate practically complete suppression of multi-spin effects if $\lambda$ was kept smaller than $1 /(N-1)$ [45], in line with previous predictions [41]. In contrast to power-scaling, $\lambda$-reduction requires modification of experimental settings, however implementation is straight-forward and no changes in sample preparation are involved.

\section{Sparse labelling}

A rather obvious suggestion to suppress multi-spin effects is eliminating their root cause by the reduction of the number of spins (or the labelling degree $f$ ) in the system under study, implying some modifications during sample preparation. Spin dilution has been used already for early CW studies [63,64], and has recently been taken into consideration for PELDOR experiments $[11,48,65,66]$. For SDSL-PELDOR with sparsely labelled samples commonly a mixture of the paramagnetic MTSL spin label with its NO-acetylated diamagnetic analogue dMTSL ((1-acetoxy-2,2,5,5-tetramethyl$\delta 3$-pyrroline-3-methyl) methanethiosulfonate) is used during the labelling procedure, allowing the precise control of the percentage of paramagnetic labelling to be achieved.

A combined approach using both $\lambda$-reduction and sparse labelling to perform distance measurements of up to $16 \mathrm{~nm}$ in a tetradecamer has been reported [11]. We have recently performed a comparative study to investigate potential performance differences in sparse labelling versus $\lambda$-reduction on the heptameric and pentameric mechanosensitive membrane channels MscS and MscL, respectively [62]. Interestingly, while both approaches were able to fully suppress multi-spin effects if the product 
$\lambda f$ was kept smaller than $1 /(N-1)$ and in combination with power-scaling, the sensitivity comparison gave clear advantages for sparse labelling (Figure 1), at least in both cytosolic mutants investigated [62]. Dipolar dephasing studies further demonstrated the detrimental effect of high local spin concentration on sensitivity. It can be rationalised that sparse labelling reduces instantaneous diffusion [67] in detection and background, while $\lambda$-reduction only reduces background decay rates; however, differences in overall modulation depths are currently under further investigation.

A

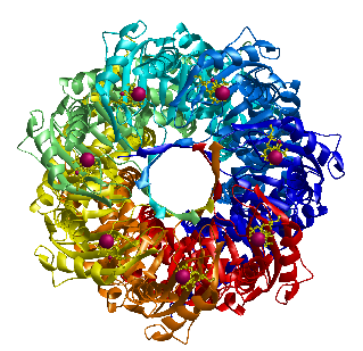

C

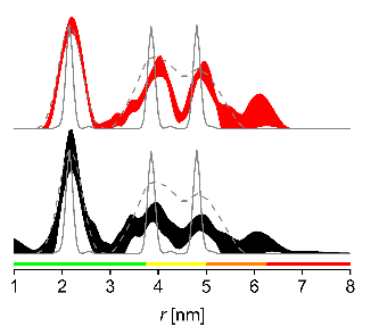

E

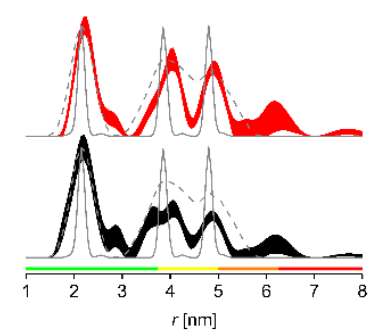

B

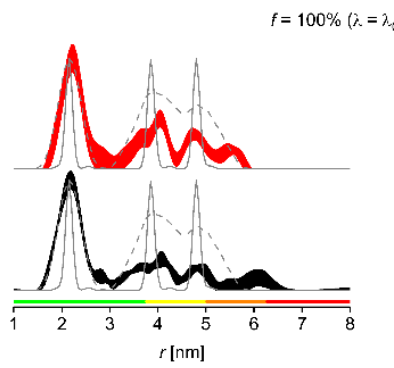

$\mathrm{D}$

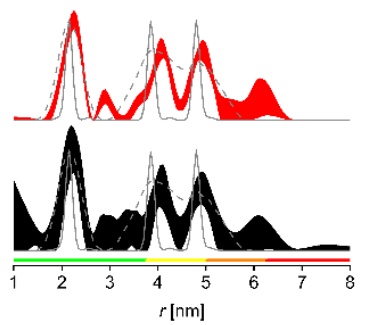

$\mathrm{F}$

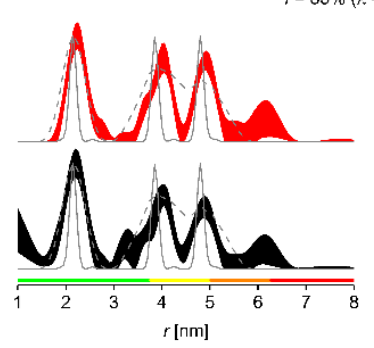

Figure 1. Comparison of $\lambda$-reduction and sparse labelling. A: MMM model of fully labeled protein. MscS S196R1 PELDOR distance distributions obtained for fully labeled protein (B), $\lambda$-reduced fully labeled protein $\left(C: \lambda=67 \% \lambda_{0}, \mathrm{D}: \lambda=33 \% \lambda_{0}\right)$, and sparsely labeled protein (E: $f=67 \%, \mathrm{~F}: f=33 \%$ ). Depicted are the $\pm 2 \sigma$ confidence intervals as black and red area for non-scaled and power-scaled data, respectively. Predicted distance distributions from MMM2015.1 and MtsslWizard are shown as gray lines (solid and dashed, respectively). The bottom color bars represent reliability ranges 
for the distance distributions as indicated in DeerAnalysis2015 (green: shape reliable; yellow: mean and width reliable; orange: mean reliable; red: no quantification possible). This figure has been published in [62] (see also for additional details) and was reproduced with permission under CC-BY license.

Technically, specific double-labelling of membrane protein complexes can be a difficult task, since the methods usually applied require either systems exchanging monomers, or systems that can be re-constituted after denaturation. Both is often not the case for membrane proteins, therefore alternative approaches are required, such as heterologous co-expression of wild-type and mutant protein [68]. For pentameric MscL, the controlled generation of heteropentamers by detergent exchange and chromatofocussing has been described recently [69], however this approach awaits generalisation.

\section{Alternative spin labels}

An entirely different approach can be taken by switching from nitroxide radicals to spin labels showing intrinsically low multi-spin effects. These have been demonstrated for high-spin paramagnetic metal ions, such as manganese or gadolinium labels [26,70]. However, these come at the cost of reduced modulation depth in the standard PELDOR experiment. Combination of these spin labels with emerging methods such as RIDME or the use of broadband excitation in PELDOR with shaped pulses could facilitate their more wide-spread application [71].

\section{Distance resolution}

Another main challenge for the determination of precise distance distributions in multimeric systems in addition to multi-spin effects is the distance resolution. The length of dipolar time domain evolution $\left(t_{\mathrm{max}}\right)$ recorded determines the frequency 
resolution. The latter limits the distance determination twofold. First, the frequency resolution determines the longest possible distance that can be reliably determined, and secondly it determines whether resolution between populations at different distances present in the system is achievable. Thus, consideration of the distance resolution will be important for correct interpretation of distance distributions beyond mean and width of the main population.

DeerAnalysis colour codes regions of the distance distribution, thereby judging the reliability of the distribution shape. In dependence of $t_{\max }$ reliability ranges will render the shape, the mean and width, or merely the mean of the distribution reliable (corresponding to a maximum distance in nm of about 3,4 , or $5 \times\left(\frac{t_{\max }}{2[\mu s]}\right)^{1 / 3}$, respectively). A validation tool allows systematically varying processing parameters and performing statistical analysis to calculate confidence bands [44,72]. Similar concepts have been used for the estimation of uncertainties in PELDOR distance distributions for other approaches to data analysis. $[73,74]$.

However, data explicitly targeting reliable separation or recognition of multiple distance populations is limited. Even in the best case of background- and noise-free simulated data recovering the input distribution is not trivial in a system with multiple distances [42]. This can be experimentally shown by use of a chemical model system with three very defined distance populations all within $1 \mathrm{~nm}$ (Figure 2). The PELDOR time trace was analysed using Tikhonov regularization in DeerAnalysis and data were truncated at different times to emulate different achievable $t_{\max }$ in challenging applications. A set of $t_{\max }$ were chosen as 1, 2, 3, or 4.6 multiples of the inverse dipolar constant $D_{\text {dip }}$ for the longest distance present $(5.2 \mathrm{~nm})$. The longest $t_{\max }$ was chosen because here, the entire distance distribution lies within the green reliability range of DeerAnalysis. Thus, the entire shape of the distribution should be reliable. Data further 
demonstrate that distance resolution is not sufficient using $t_{\max }=2 / D_{\text {dip }}$, even with excellent experimental signal-to-noise (here above 400 to 1 ).

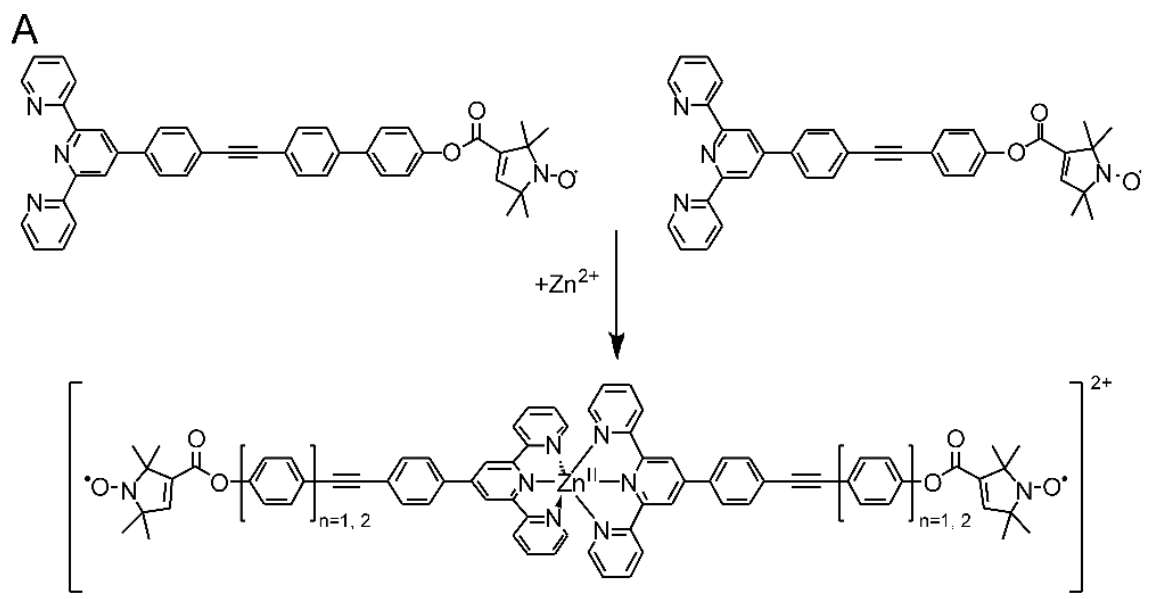

B

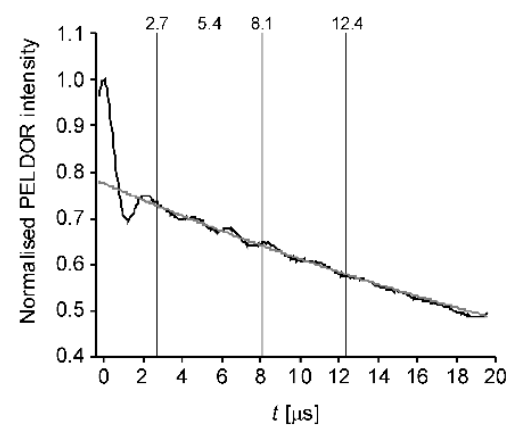

D

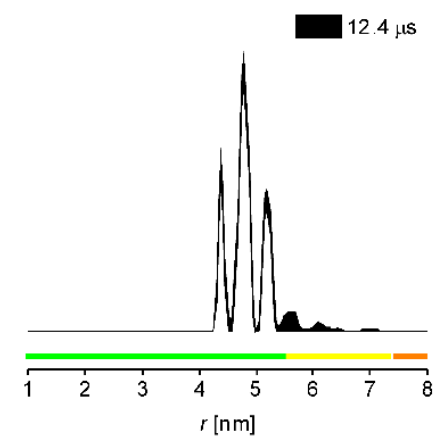

$\mathrm{F}$

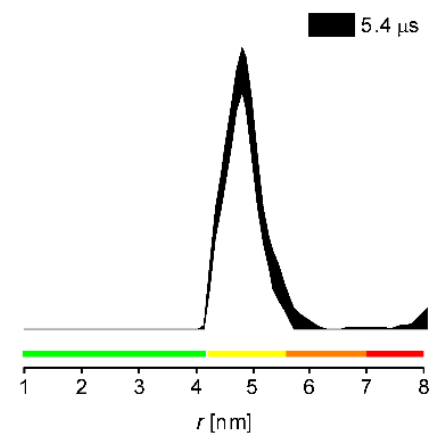

C

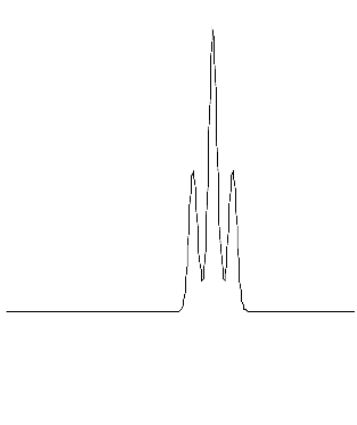

$E$

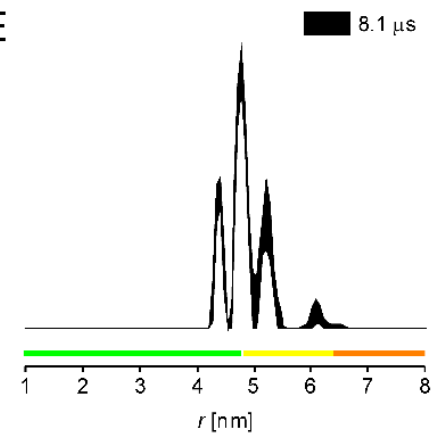

G

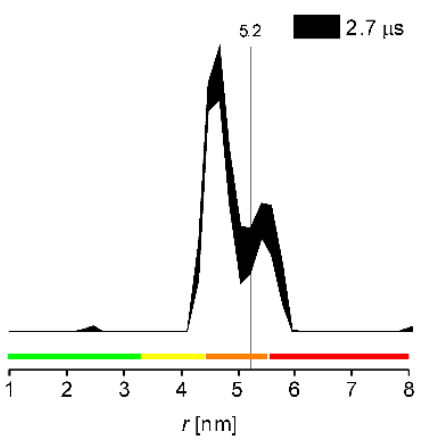

Figure 2. Distance resolution. A: Two terpyridine-based compounds are used for templated complex formation with $\mathrm{Zn}^{2+}$ as the template. Dimerisation on the template occurs with a statistical distribution of possible complexes; a model distance 
distribution is shown in C. B: PELDOR trace of the resulting mix of dimers; vertical lines indicate dipolar oscillation times corresponding to $1,2,3$, or 4.6 multiples of the inverse dipolar coupling corresponding to the longest distance present $(5.2 \mathrm{~nm})$, these were used for data truncation before Tikhonov regularisation. D-G: Distance distributions with $\pm 2 \sigma$ confidence intervals obtained by statistical analysis. The vertical line in G corresponds to the expected cut-off distance (at $\left.r / \mathrm{nm}=(t / \mu \mathrm{s} \times 52)^{1 / 3}\right)$ and is shown to highlight the right peak as an artefact. The color bars represent reliability ranges for the distance distributions as indicated in DeerAnalysis2015 (green: shape reliable; yellow: mean and width reliable; orange: mean reliable; red: no quantification possible). More details on distance resolution and the synthesis and characterization of the shorter terpyridine ligand will be published elsewhere.

Furthermore, measuring at even shorter dipolar evolution times often leads to artefact peaks as the background correction (eq 4) becomes unreliable. Such artificial distance populations could easily be misinterpreted as a true peak since they are often rendered significant by validation of the data by statistical analyses over a systematically varied background correction. A detailed study on the resolution of multiple distances is ongoing and will be reported elsewhere.

\section{Multimerisation studies - quantitative assessment of the modulation depth $\Delta$}

\section{PELDOR}

In addition to the dipolar oscillation encoding the distance in a PELDOR experiment, the modulation depth $\Delta$ contains information about the number of spins in the system and thus, the multimerisation degree [21]. Furthermore, $\Delta$ can be used to follow multimerisation equilibria such as the liner build-up of modulation depth upon increased dimer formation. Quantitative assessment of changes in $\Delta$ allows determining binding constants [75]. 
Several PELDOR studies have demonstrated the usefulness of quantitative assessment of $\Delta$ in biological systems. The true dimer interface of the influenza A virus non-structural protein 1 (NS1) could be identified based on changes in $\Delta$ employing a mutant known to inhibit dimerisation [76], and incomplete dimerisation of the effector domain could be concluded from a reduced $\Delta$ [76], in agreement with weak binding observed using ${ }^{19} \mathrm{~F}$ NMR [77]. In another PELDOR study multimerisation of the influenza A M2 transmembrane domain could be assessed [78]. We recently demonstrated that $\Delta$ behaves as expected in the pentameric mechanosensitive channel of large conductance from E. coli (MscL) [62]. Only in higher oligomers, such as the $E$. coli heptameric mechanosensitive channel of small conductance $(\mathrm{MscS})$ lower values than expected were reported $[15,45]$, indicating that in these systems other effects might take over.

\section{RIDME}

In contrast to PELDOR, RIDME is a single frequency technique [32,79]. While in a PELDOR experiment the bandwidth of the pump pulse is limiting, this is not the case for relaxation-based RIDME, which has been shown to result in a larger modulation depth and thus, sensitivity when pulse bandwidth is limiting [80]. This makes RIDME especially attractive in combination with paramagnetic metal ions and could prove highly useful for studying proteins with native or engineered metal binding sites.

RIDME is still an emerging technique, and quantitative assessment of the modulation depth $\Delta$ for multimerisation studies has not yet been demonstrated in a biological system. However, recently it was shown in a templated dimerisation model system that dimerisation can be followed using $\Delta$, similarly to PELDOR $[75,81]$. 
However, applicability to challenging biomolecular systems remains to be demonstrated.

For multimerisation studies on higher oligomers bearing multiple metal centres (i.e., in a multi-spin system), multi-spin effects are expected to come into play using RIDME similarly to PELDOR on nitroxides. It will be very useful to see if RIDME will allow determining higher oligomerisation degrees and whether the different approaches for recovering the true distance distribution will be transferable from PELDOR to RIDME.

\section{Conclusion and Perspectives}

PD-EPR distance measurements have become an important tool in the armoury of structural biology methods. However, applications to complex biological systems such as multimeric membrane proteins have exposed complications arising from multi-spin effects and the presence of multiple distances. Substantial advances have been made in overcoming these challenges, and the use of PELDOR in combination with powerscaling, sparse labelling and/or $\lambda$-reduction will have extended the scope of the method to multimeric systems which become tractable with careful experimental design.

In addition, PD-EPR methods have been demonstrated to be highly suited to assess the multimerisation degree and to determine binding constants using the modulation depths. Initial model studies will have to be translated to complex biomolecules as has been shown for PELDOR [78]. For RIDME as an emerging, highly promising technique for measurements involving fast relaxing paramagnetic metal ions, these applications will widen the information that can be retrieved and thus, the scope of applications. 
Studying systems with multiple distances using PD-EPR requires both, accuracy and precision, becoming particularly challenging in combination with additional multispin issues in multimers. A good understanding of the achievable distance resolution and in turn its limits is highly important. More research into the reliable separation of multiple distance peaks of different widths and intensities is in progress.

\section{Acknowledgements}

This work was supported by funding from the European Union (Marie Curie Actions REA 334496), the Carnegie Trust (70098), the EPSRC (EP/M024660/1) and a Wellcome Trust multiuser equipment grant (099149/Z/12/Z).

\section{References}

[1] G. Jeschke, Annu. Rev. Phys. Chem. 63, 419 (2012).

[2] O. Schiemann, and T.F. Prisner, Q. Rev. Biophys. 40, 1 (2007).

[3] R.E. Martin, M. Pannier, F. Diederich, V. Gramlich, M. Hubrich, and H.W. Spiess, Angew. Chem., Int. Ed. 37, 2834 (1998).

[4] A.D. Milov, K.M. Salikov, and M.D. Shirov, Fiz. Tverd. Tela 23, 975 (1981).

[5] M. Pannier, S. Veit, A. Godt, G. Jeschke, and H.W. Spiess, J. Magn. Reson. 142, 331 (2000).

[6] S. Saxena, and J.H. Freed, Chem. Phys. Lett. 251, 102 (1996).

[7] C. Altenbach, S.L. Flitsch, H.G. Khorana, and W.L. Hubbell, Biochemistry 28, 7806 (1989).

[8] W.L. Hubbell, and C. Altenbach, Curr. Opin. Struct. Biol. 4, 566 (1994).

[9] A. Bowman, C.M. Hammond, A. Stirling, R. Ward, W. Shang, H. El-Mkami, D.A. Robinson, D.I. Svergun, D.G. Norman, and T. Owen-Hughes, Nucleic Acids Res. 42, 6038 (2014).

[10] G. Jeschke, eMagRes 5, 1459 (2016).

[11] T. Schmidt, M.A. Walti, J.L. Baber, E.J. Hustedt, and G.M. Clore, Angew. Chem., Int. Ed. 55, 15905 (2016).

[12] O. Duss, E. Michel, M. Yulikov, M. Schubert, G. Jeschke, and F.H. Allain, Nature 509, 588 (2014).

[13] B. Verhalen, R. Dastvan, S. Thangapandian, Y. Peskova, H.A. Koteiche, R.K. Nakamoto, E. Tajkhorshid, and H.S. McHaourab, Nature 543, 738 (2017).

[14] C. Pliotas, A.C. Dahl, T. Rasmussen, K.R. Mahendran, T.K. Smith, P. Marius, J. Gault, T. Banda, A. Rasmussen, S. Miller, C.V. Robinson, H. Bayley, M.S. Sansom, I.R. Booth, and J.H. Naismith, Nat. Struct. Mol. Biol. 22, 991 (2015).

[15] C. Pliotas, R. Ward, E. Branigan, A. Rasmussen, G. Hagelueken, H.X. Huang, S.S. Black, I.R. Booth, O. Schiemann, and J.H. Naismith, Proc. Natl. Acad. Sci. U. S. A. 109, E2675 (2012).

[16] S.Y. Park, P.P. Borbat, G. Gonzalez-Bonet, J. Bhatnagar, A.M. Pollard, J.H. Freed, A.M. Bilwes, and B.R. Crane, Nat. Struct. Mol. Biol. 13, 400 (2006). 
[17] B. Carrington, W.K. Myers, P. Horanyi, M. Calmiano, and A.D.G. Lawson, Biophys. J. 113, 371 (2017).

[18] R.G. Larsen, and D.J. Singel, J. Chem. Phys. 98, 5134 (1993).

[19] G. Jeschke, M. Sajid, M. Schulte, and A. Godt, Phys. Chem. Chem. Phys. 11, 6580 (2009).

[20] A.D. Milov, and Y.D. Tsvetkov, Appl. Magn. Reson. 12, 495 (1997).

[21] B.E. Bode, D. Margraf, J. Plackmeyer, G. Dürner, T.F. Prisner, and O. Schiemann, J. Am. Chem. Soc. 129, 6736 (2007).

[22] A.D. Milov, A.B. Ponomarev, and Y.D. Tsvetkov, Chem. Phys. Lett. 110, 67 (1984).

[23] W.L. Hubbell, C.J. Lopez, C. Altenbach, and Z. Yang, Curr. Opin. Struct. Biol. 23, 725 (2013).

[24] B.E. Bode, J. Plackmeyer, T.F. Prisner, and O. Schiemann, J. Phys. Chem. A 112, 5064 (2008).

[25] T.F. Cunningham, M.R. Putterman, A. Desai, W.S. Horne, and S. Saxena, Angew. Chem., Int. Ed. 54, 6330 (2015).

[26] A. Dalaloyan, M. Qi, S. Ruthstein, S. Vega, A. Godt, A. Feintuch, and D. Goldfarb, Phys. Chem. Chem. Phys. 17, 18464 (2015).

[27] M. Ji, S. Ruthstein, and S. Saxena, Acc. Chem. Res. 47, 688 (2014).

[28] I. Krstic, R. Hansel, O. Romainczyk, J.W. Engels, V. Dotsch, and T.F. Prisner, Angew. Chem., Int. Ed. 50, 5070 (2011).

[29] A. Martorana, G. Bellapadrona, A. Feintuch, E. Di Gregorio, S. Aime, and D. Goldfarb, J. Am. Chem. Soc. 136, 13458 (2014).

[30] P.E. Spindler, S.J. Glaser, T.E. Skinner, and T.F. Prisner, Angew. Chem., Int. Ed. 52, 3425 (2013).

[31] P.E. Spindler, P. Schops, A.M. Bowen, B. Endeward, and T.F. Prisner, eMagRes 5, 1477 (2016).

[32] L.V. Kulik, S.A. Dzuba, I.A. Grigoryev, and Y.D. Tsvetkov, Chem. Phys. Lett. 343, 315 (2001).

[33] S. Milikisyants, F. Scarpelli, M.G. Finiguerra, M. Ubbink, and M. Huber, J. Magn. Reson. 201, 48 (2009).

[34] K. Keller, A. Doll, M.A. Qi, A. Godt, G. Jeschke, and M. Yulikov, J. Magn. Reson. 272, 108 (2016).

[35] K. Keller, V. Mertens, M. Qi, A.I. Nalepa, A. Godt, A. Savitsky, G. Jeschke, and M. Yulikov, Phys. Chem. Chem. Phys. 19, 17856 (2017).

[36] T. Wiegand, D. Lacabanne, K. Keller, R. Cadalbert, L. Lecoq, M. Yulikov, L. Terradot, G. Jeschke, B.H. Meier, and A. Bockmann, Angew. Chem., Int. Ed. 56, 3369 (2017).

[37] M.D. Rabenstein, and Y.K. Shin, Proc. Natl. Acad. Sci. U. S. A. 92, 8239 (1995).

[38] J. Pyka, J. Ilnicki, C. Altenbach, W.L. Hubbell, and W. Froncisz, Biophys. J. 89, 2059 (2005).

[39] D. Marsh, Proc. Natl. Acad. Sci. U. S. A. 98, 7777 (2001).

[40] E. Perozo, D.M. Cortes, P. Sompornpisut, A. Kloda, and B. Martinac, Nature 418, 942 (2002).

[41] T. von Hagens, Y. Polyhach, M. Sajid, A. Godt, and G. Jeschke, Phys. Chem. Chem. Phys. 15, 5854 (2013).

[42] A. Giannoulis, R. Ward, E. Branigan, J.H. Naismith, and B.E. Bode, Mol. Phys. 111, 2845 (2013).

[43] Y.W. Chiang, P.P. Borbat, and J.H. Freed, J. Magn. Reson. 172, 279 (2005). 
[44] G. Jeschke, V. Chechik, P. Ionita, A. Godt, H. Zimmermann, J. Banham, C.R. Timmel, D. Hilger, and H. Jung, Appl. Magn. Reson. 30, 473 (2006).

[45] S. Valera, K. Ackermann, C. Pliotas, H. Huang, J.H. Naismith, and B.E. Bode, Chem. - Eur. J. 22, 4700 (2016).

[46] H.S. Mchaourab, P.R. Steed, and K. Kazmier, Structure 19, 1549 (2011).

[47] D. Dawidowski, and D.S. Cafiso, Biophys. J. 104, 1585 (2013).

[48] D.T. Edwards, T. Huber, S. Hussain, K.M. Stone, M. Kinnebrew, I. Kaminker,

E. Matalon, M.S. Sherwin, D. Goldfarb, and S. Han, Structure 22, 1677 (2014).

[49] B. Endeward, J.A. Butterwick, R. MacKinnon, and T.F. Prisner, J. Am. Chem. Soc. 131, 15246 (2009).

[50] E.R. Georgieva, P.P. Borbat, C. Ginter, J.H. Freed, and O. Boudker, Nat. Struct. Mol. Biol. 20, 215 (2013).

[51] G. Hagelueken, W.J. Ingledew, H. Huang, B. Petrovic-Stojanovska, C.

Whitfield, H. ElMkami, O. Schiemann, and J.H. Naismith, Angew. Chem., Int. Ed. 48, 2904 (2009).

[52] U.A. Hellmich, S. Lyubenova, E. Kaltenborn, R. Doshi, H.W. van Veen, T.F. Prisner, and C. Glaubitz, J. Am. Chem. Soc. 134, 5857 (2012).

[53] D. Hilger, H. Jung, E. Padan, C. Wegener, K.P. Vogel, H.J. Steinhoff, and G. Jeschke, Biophys. J. 89, 1328 (2005).

[54] G. Jeschke, C. Wegener, M. Nietschke, H. Jung, and H.J. Steinhoff, Biophys. J. 86, 2551 (2004).

[55] B. Joseph, A. Sikora, E. Bordignon, G. Jeschke, D.S. Cafiso, and T.F. Prisner, Angew. Chem., Int. Ed. 54, 6196 (2015).

[56] K. Kazmier, S. Sharma, S.M. Islam, B. Roux, and H.S. McHaourab, Proc. Natl. Acad. Sci. U. S. A. 111, 14752 (2014).

[57] R. Langen, J.M. Isas, W.L. Hubbell, and H.T. Haigler, Proc. Natl. Acad. Sci. U. S. A. 95, 14060 (1998).

[58] M.C. Puljung, H.A. DeBerg, W.N. Zagotta, and S. Stoll, Proc. Natl. Acad. Sci. U. S. A. 111, 9816 (2014).

[59] F. Rodriguez, S.L. Rouse, C.E. Tait, J. Harmer, A. De Riso, C.R. Timmel, M.S. Sansom, B.C. Berks, and J.R. Schnell, Proc. Natl. Acad. Sci. U. S. A. 110, E1092 (2013).

[60] K.M. Stone, J. Voska, M. Kinnebrew, A. Pavlova, M.J. Junk, and S. Han, Biophys. J. 104, 472 (2013).

[61] Q. Xu, J.F. Ellena, M. Kim, and D.S. Cafiso, Biochemistry 45, 10847 (2006).

[62] K. Ackermann, C. Pliotas, S. Valera, J.H. Naismith, and B.E. Bode, Biophys. J. 113, 1968 (2017).

[63] R. Langen, J.M. Isas, H. Luecke, H.T. Haigler, and W.L. Hubbell, J. Biol. Chem. 273, 22453 (1998).

[64] E. Perozo, D.M. Cortes, and L.G. Cuello, Nat. Struct. Biol. 5, 459 (1998).

[65] M. Aitha, T.K. Richmond, Z. Hu, A. Hetrick, R. Reese, A. Gunther, R. McCarrick, B. Bennett, and M.W. Crowder, J. Inorg. Biochem. 136, 40 (2014).

[66] M.J. Junk, H.W. Spiess, and D. Hinderberger, J. Magn. Reson. 210, 210 (2011).

[67] J.R. Klauder, and P.W. Anderson, Phys. Rev. 125, 912 (1962).

[68] J.J. Kerrigan, Q. Xie, R.S. Ames, and Q. Lu, Protein Expr. Purif. 75, 1 (2011).

[69] D. Yilmaz, A.I. Dimitrova, M. Walko, and A. Kocer, Eur. Biophys. J. 44, 557 (2015).

[70] D. Goldfarb, Struct. Bond. 152, 163 (2012).

[71] C. Gmeiner, G. Dorn, F.H.T. Allain, G. Jeschke, and M. Yulikov, Phys. Chem. Chem. Phys., DOI: 10.1039/c7cp05822e (2017). 
[72] G. Jeschke, Struct. Bond. 152, 83 (2014).

[73] T.H. Edwards, and S. Stoll, J. Magn. Reson. 270, 87 (2016).

[74] R.A. Stein, A.H. Beth, and E.J. Hustedt, Methods Enzymol. 563, 531 (2015).

[75] K. Ackermann, A. Giannoulis, D.B. Cordes, A.M. Slawin, and B.E. Bode, Chem. Commun. 51, 5257 (2015).

[76] P.S. Kerry, H.L. Turkington, K. Ackermann, S.A. Jameison, and B.E. Bode, J. Phys. Chem. B 118, 10882 (2014).

[77] J.M. Aramini, K. Hamilton, L.C. Ma, G.V. Swapna, P.G. Leonard, J.E. Ladbury, R.M. Krug, and G.T. Montelione, Structure 22, 515 (2014).

[78] E.R. Georgieva, P.P. Borbat, H.D. Norman, and J.H. Freed, Sci. Rep. 5, 11757 (2015).

[79] A.V. Astashkin, Methods Enzymol. 563, 251 (2015).

[80] A. Collauto, V. Frydman, M.D. Lee, E.H. Abdelkader, A. Feintuch, J.D. Swarbrick, B. Graham, G. Otting, and D. Goldfarb, Phys. Chem. Chem. Phys. 18, 19037 (2016).

[81] A. Giannoulis, M. Oranges, and B.E. Bode, ChemPhysChem 18, 2318 (2017). 